\title{
Comparison between cone-beam and multislice computed tomography for identification of simulated bone lesions
}

\author{
Bruno Felipe Gaia(a) \\ Marcelo Augusto Oliveira \\ de Sales ${ }^{(b)}$ \\ Andréia Perrella ${ }^{(c)}$ \\ Marlene Fenyo-Pereira ${ }^{(d)}$ \\ Marcelo Gusmão Paraíso \\ Cavalcanti ${ }^{(d)}$ \\ (a) Stomatology Department, Dental School, \\ University of São Paulo, São Paulo, SP, \\ Brazil. \\ (b) Department of Radiology, Dental School, \\ University of Paraíba, João Pessoa, PB, \\ Brazil. \\ (c) Private practice, São Paulo, SP, Brazil. \\ (d) Department of Radiology, Dental School, \\ University of São Paulo, São Paulo, SP, \\ Brazil.
}

\begin{abstract}
There are many studies that compare the accuracy of multislice (MSCT) and cone beam (CBCT) computed tomography for evaluations in the maxillofacial region. However, further studies comparing both acquisition techniques for the evaluation of simulated mandibular bone lesions are needed. The aim of this study was to compare the accuracy of MSCT and CBCT in the diagnosis of simulated mandibular bone lesions by means of cross sectional images and axial/MPR slices. Lesions with different dimensions, shape and locularity were produced in 15 dry mandibles. The images were obtained following the cross sectional and axial/MPR (Multiplanar Reconstruction) imaging protocols and were interpreted independently. CBCT and MSCT showed similar results in depicting the percentage of cortical bone involvement, with great sensitivity and specificity $(\mathrm{p}<0.005)$. There were no significant intra- or inter-examiner differences between axial/MPR images and cross sectional images with regard to sensitivity and specificity. CBCT showed results similar to those of MSCT for the identification of the number of simulated bone lesions. Cross sectional slices and axial/MPR images presented high accuracy, proving useful for bone lesion diagnosis.
\end{abstract}

Descriptors: Cone-Beam Computed Tomography; Tomography, Spiral Computed; Mandible.

\section{Introduction}

Rapid development of computer technology, improved performance, accessibility and lower cost have pathed the way for digital and advanced imaging modalities. Computed tomography (CT) images provide important information regarding the individual characteristics of pathological lesions, information which is particularly useful for diagnoses and treatments. ${ }^{1}$ The introduction of multislice computed tomography (MSCT) represented a fundamental evolutionary step in the development and ongoing refinement of CT imaging techniques. ${ }^{2} \mathrm{~A}$ single MSCT scan can yield multiple, thin, overlapping slices that can be rapidly reconstructed, resulting in higher-quality reconstructed images and precluding the need for further patient radiation exposure. ${ }^{3}$

Cone-beam computed tomography (CBCT) stands out as an alternative to MSCT. This recently-designed technology became a relevant tool for oral and maxillofacial diagnostic osseous imaging, providing to professionals access to excellent image quality and greater diagnostic ac-
Received for publication on Apr 19, 2011 Accepted for publication on Jun 09, 2011

\section{Corresponding author:}

Marcelo Gusmão Paraíso Cavalcanti

E-mail:mgpcaval@usp.br 
curacy and sensitivity. ${ }^{4,5}$ In addition, CBCT allows images to be acquired using a low dose of radiation, shorter patient examination time, and lower costs than MSCT, which makes its routine use feasible for oral and maxillofacial procedures. ${ }^{6,7}$ It has been demonstrated that MSCT and CBCT are useful for evaluations in the oral and maxillofacial regions, ${ }^{8-11}$ although further studies are necessary comparing both acquisition techniques for the evaluation of bone lesions.

Several studies have reported that the interpretation allowed by different CT imaging modalities supply more information than axial images, aiding in achieving more reliable diagnosis and effective therapeutics. Furthermore, multiplanar reconstruction (MPR) and cross sectional images provide improvements in the diagnosis, rehabilitation and evaluation of bone pathological processes in the maxillofacial complex. ${ }^{12-14}$

The purpose of this research was to compare the accuracy of MSCT and CBCT in the diagnosis of simulated mandibular bone lesions by means of cross sectional images and axial/MPR slices.

\section{Methodology}

The present study was submitted to and approved by the Committee of Ethics and Research of our Institution, under protocol \# 151/2003.

A total of 15 dry mandibles were examined. Lesions involving cortical bone were produced using a round bur (H1, number 8, Komet Brasil, Santo André, Brazil) and a high-speed handpiece (Silent MS 350PB, Dabi Atlante, Ribeirão Preto, Brazil). The lesions, which differed in dimension and shape, were produced either in the buccal cortical bone or in the lingual cortical bone of the mandibular body. In some cases, the bur just touched the cortical bone, whereas in others, it perforated the medullary bone. A total of 52 perforations were made, ranging in diameter from $1 \mathrm{~mm}$ to $3 \mathrm{~mm}$ and in depth from $0.5 \mathrm{~mm}$ to $3.0 \mathrm{~mm}$. In 7 mandibles, unilocular lesions were produced and, in 8 mandibles, multilocular lesions (having 3 to 9 loculi each) were produced on the lingual surface of the body of the mandible. In 2 of these mandibles, the buccal cortical bone of the mandibular body was perforated (Figure 1).
The mandibles were submitted to a CBCT scanner (i-CAT ${ }^{\circledast}$ Cone Beam 3-D Dental Imaging System; Imaging Sciences International, Hatfield, USA) with the following parameters: voxel size of $0.25 \mathrm{~mm}$; raw data acquisition of $40 \mathrm{~s}$; exposure settings of $90 \mathrm{kVp}$ and $7 \mathrm{~mA}$; and a display field of view of $15 \mathrm{~cm}$. Subsequently, MSCT was performed (Aquilion 64; Toshiba Medical Systems, Tustin, USA) with the following parameters: slice thickness of $0.5 \mathrm{~mm}$; reconstruction interval of $0.3 \mathrm{~mm}$; exposure time of $0.4 \mathrm{~s}(120 \mathrm{kVp}, 300 \mathrm{~mA}$ and $512 \times 512$ pixel matrix); bone tissue filter; and a field of view of $18 \mathrm{~cm}$.

For image acquisition, the specimens were placed in a plastic bucket, completely covered with water (in order to simulate soft tissue) and maintained in the same position as that used in in vivo studies (using cotton sheets for support). Axial slices were acquired, the specimens being scanned from the mandibular base to the condyle. The scanning plane was parallel to the mandibular base. Gantry angles varied according to mandibular base angles.

The original data were sent to a workstation. The association of MPR and axial images (with simultaneous analysis of coronal and sagittal views) were displayed and analyzed using Vitrea ${ }^{\circledR}$ software, version 3.4.5 (Vital Images Inc., Plymouth, USA), and the cross sectional images were analyzed using Imaging Studio ${ }^{\circledR}$ software, version 2.556 (Anne Solutions, São Paulo, Brazil). Both protocols were interpreted independently by two experienced examiners (oral and maxillofacial radiologists). The analyses of

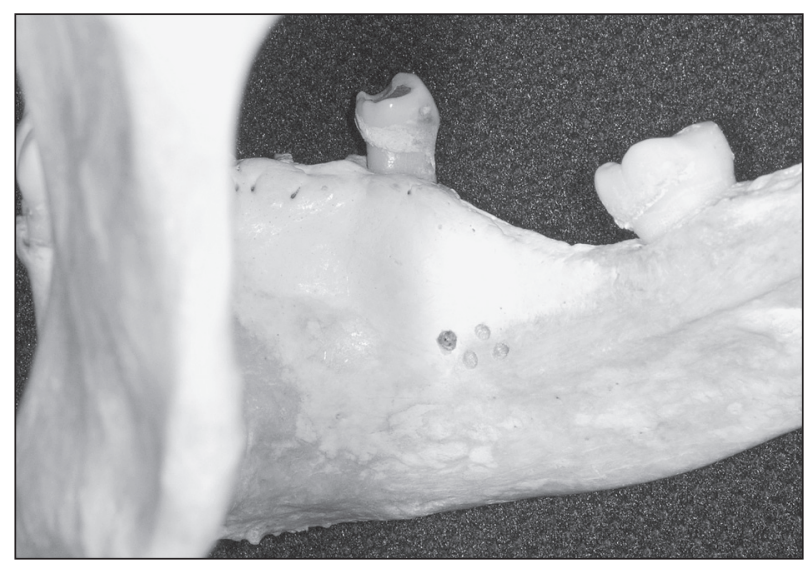

Figure 1 - Simulated bone lesions on the lingual cortical bone of the mandibular body. 

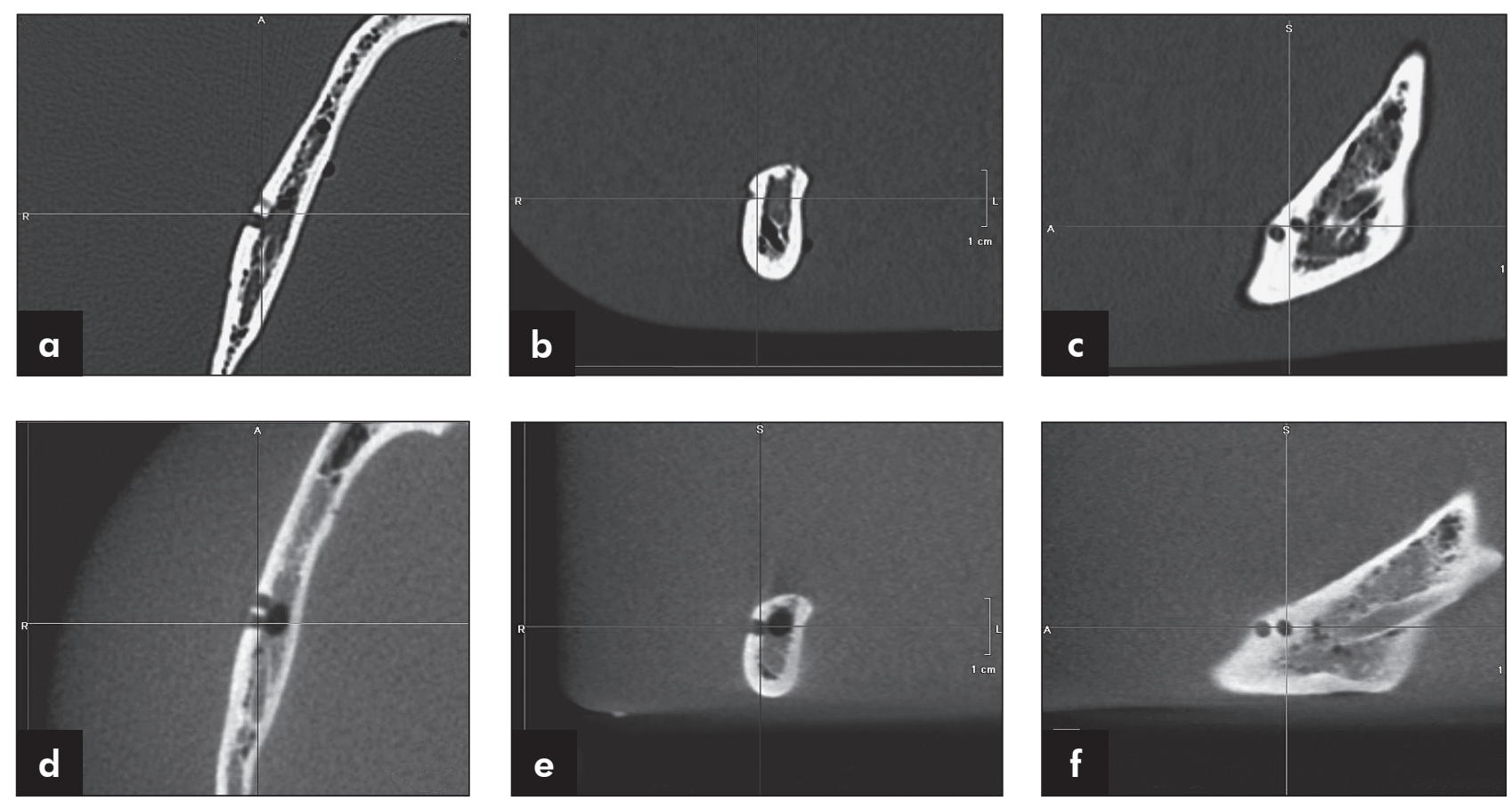

Figure 2 - $M S C T(\mathbf{a}, \mathbf{b}, \mathbf{c})$ and $C B C T(\mathbf{d}, \mathbf{e}, \mathbf{f})$ axial/MPR images.

the images were performed in a random order of the protocols, in different sessions (with an interval of at least two weeks between sessions). The examiners had no contact with the specimens and were blinded to the acquisition technique used and to the characteristics of the lesions in each mandible.

The examiners were asked to identify the different characteristics of the lesions (whether the cortical bone had been perforated and the number of lesions in each mandible) in each protocol. The perforations in the dry mandibles were considered the gold standard.

During the analysis of the images, only the protocol images were displayed on the computer monitor in order not to influence the interpretation (Figures 2 and 3 ).

Intra- and inter-examiner reliability was calculated using the kappa statistic, the validity test (sensitivity and specificity) and the chi-square test. The validity test is represented by the Youden index, which attempts to represent test accuracy by a single numerical value (sensitivity + specificity -1$)$. The program Statistical Package for the Social Sciences, version 12.0 for Windows (SPSS Inc., Chicago, USA) was used. A 95\% ( $<<0.05)$ confidence inter-

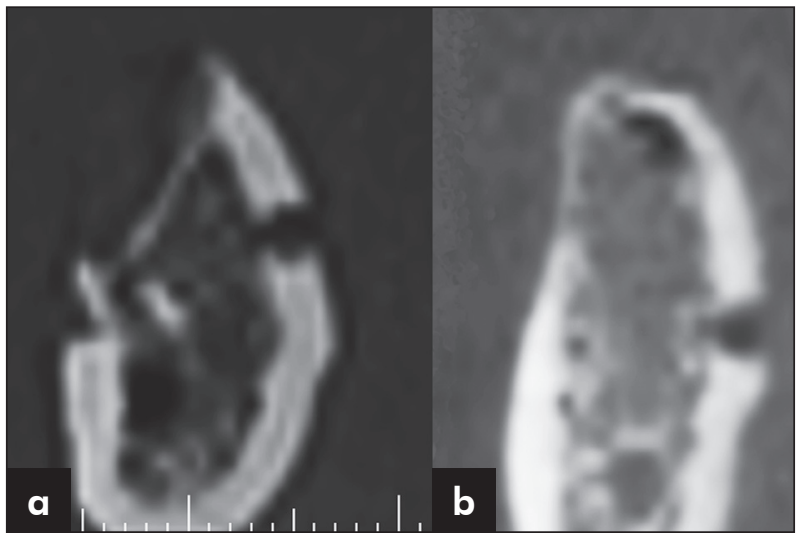

Figure 3 - MSCT (a) and CBCT (b) cross sectional images.

val was used.

\section{Results}

Results regarding the detection of the number of lesions showed high agreement between examiners, as shown in Table 1.

The number of bone lesions was related to the interpretation criteria used for the detection of simulated bone lesions (absence, unilocular or multilocular lesion), according to the protocol used and the gold standard. 
The data collected showed that both examiners were able to identify simulated mandibular lesions using MSCT and CBCT following both protocols, with no statistical difference between them (over $95 \%$ of the lesions were identified) (Table 1 ). The analyses involving the protocols (axial/MPR and cross sectional slices) were highly effective in aiding both examiners in identifying the number of lesions.

The kappa statistic was used to assess agreement between examiners. In the sample as a whole, a kappa value of 0.869 was found between MSCT and CBCT in the overall evaluation. In addition, the Kappa statistic was used for an individual evaluation of the protocols, comparing the results obtained by examiner 1 , examiner 2 , and the gold standard (Table 2).

Table 1 - Frequency of detection of simulated bone lesions.

\begin{tabular}{c|c|c|c}
\hline & Examiner 1 & Examiner 2 & Gold Standard \\
\hline Absence & 348 & 351 & 380 \\
\hline Unilocular & 317 & 309 & 290 \\
\hline Multilocular & 235 & 240 & 230 \\
\hline Total & 900 & 900 & 900 \\
\hline
\end{tabular}

The protocols were statistically significant for the intra- and inter-examiner analyses regarding the number of lesions, which proved the validity of the methods.

As regards the sensitivity and specificity in detecting the number of mandibular lesions, the association between axial slices and MPR showed better results than the cross sectional images, with no significant intra- or inter-examiner differences (Table 3).

MSCT and CBCT showed excellent results for both protocols, with no significant intra- or interexaminer differences.

\section{Discussion}

Imaging of the maxillofacial region is limited with conventional radiography due to the overlap of anatomical structures, making visualization of the area of interest very difficult. ${ }^{8,15}$ MSCT and CBCT represent an important advance because, using these methods, observers can reconstruct and manipulate high resolution images, thus improving the diagnosis. ${ }^{15,16}$

Both modalities of image acquisition have advantages and disadvantages regarding radiation dose,
Table 2 - Kappa and $p$ values for the comparison between examiner 1 , examiner 2 , and gold standard for the identification of the number of simulated bone lesions.

\begin{tabular}{c|c|c|c|c|c|c}
\hline & \multicolumn{5}{|c}{ Number of cavities } \\
\cline { 2 - 7 } & Examiner 1 X Examiner 2 & Examiner 1 X Gold Standard & \multicolumn{2}{c}{ Examiner 2 X Gold Standard } \\
\cline { 2 - 7 } & Kappa & $p$ & Kappa & $p$ & Kappa & $p$ \\
\hline $\begin{array}{c}\text { MSCT } \\
\text { MPR/axial }\end{array}$ & 0.935 & $<0.005$ & 0.951 & $<0.005$ & 0.886 & $<0.005$ \\
\hline $\begin{array}{c}\text { MSCT } \\
\text { parasagittal }\end{array}$ & 0.838 & $<0.005$ & 0.855 & $<0.005$ & 0.886 & $<0.005$ \\
\hline $\begin{array}{c}\text { CBCT } \\
\text { MPR/axial }\end{array}$ & 0.952 & $<0.005$ & 0.935 & $<0.005$ & 0.951 & $<0.005$ \\
\hline $\begin{array}{c}\text { CBCT } \\
\text { parasagittal }\end{array}$ & 0.707 & $<0.005$ & 0.803 & $<0.005$ & 0.886 & $<0.005$ \\
\hline
\end{tabular}

Table 3 - Percentage rates of detection of the number of simulated bone lesions.

\begin{tabular}{c|c|c|c|c}
\hline Examiner 1 & MSCT MPR/axial & MSCT parasagittal & CBCT MPR/axial & CBCT parasagittal \\
\hline Sensitivity & $92.6 \%$ & $86.3 \%$ & $92 \%$ & $86.6 \%$ \\
\hline Specificity & $94.5 \%$ & $94.5 \%$ & $90.4 \%$ & $97.7 \%$ \\
\hline Examiner 2 & MSCT MPR/axial & MSCT parasagittal & CBCT MPR/axial & CBCT parasagittal \\
\hline Sensitivity & $95 \%$ & $88 \%$ & $98 \%$ & $86.3 \%$ \\
\hline Specificity & $96.2 \%$ & $100 \%$ & $97.4 \%$ & $98.1 \%$ \\
\hline
\end{tabular}


acquisition time, cost, scattered radiation and artifacts. ${ }^{6,7}$ Drawbacks should be taken into consideration, since they can influence image quality and interpretation accuracy. The applicability of CT images has been broadly shown for several purposes such as craniometric measurements and anatomical identification, forensic identification, diagnosis and surgical planning of fractures, and maxillofacial implants. ${ }^{7,17}$ However, few studies have compared the accuracy of MSCT and CBCT for the identification of mandibular lesions, indicating the need for conducting new studies.

Simulated bone lesions have been widely used to compare radiological techniques for bone observation. ${ }^{16}$ Pinsky et al. ${ }^{18}$ used simulated lesions to test linear measurements in CBCT, but they made 4-8$\mathrm{mm}$ defects, which are larger than those prepared in the present work. In these experiments, water was added to produce an environment closer to bone in vivo. This methodological procedure was the same used in the present experiment to attenuate the $\mathrm{x}$ ray. This study used mechanical pseudo-lesions that are not radiographically identical to those developed naturally; simulated lesions were chosen in order to have a defined pattern to allow a comparison of the image acquisition modalities and the observation protocols, in order to approximate our findings to a true pathological bone lesion condition. The validity of the methods was confirmed when the results obtained by examiners 1 and 2 were compared with the gold standard (Kappa > 0.869) for identification of the number of simulated bone lesions.

Perrella et al. ${ }^{13}$ showed that the techniques based on helical acquisition were recognizably capable of providing high sensitivity and specificity in the diagnosis of simulated mandibular bone lesions with and without metallic artifacts. Mischkowski et al. ${ }^{19}$ and Dreiseidler et al. ${ }^{20}$ reported that the diagnostic value was the same between CBCT and MSCT images, both methods providing good visualization of various mandibular anatomical structures. Our study corroborated to the results of these authors in that both modalities were equally capable of detecting the presence of simulated mandibular bone lesions, showing high specificity and sensitivity values.

The importance of acquisition parameters, such as slice thickness, in bone lesion evaluation were discussed previously by many authors. ${ }^{21-23}$ Shaha ${ }^{23}$ stated that for detailed evaluation of the mandible it is essential to obtain CT scans with bone windows and narrow cuts, and the accuracy found in his work was $68 \%$. According to Baxter and Sorenson ${ }^{21}$, identification of the number of lesions was inaccurate when the diameter was comparable to or less than the CT slice thickness, not allowing a correct identification of bone lesions. Furthermore, Cavalcanti et al. ${ }^{22}$ demonstrated high false positive and false negative rates when determining mandibular bone invasion using 3-mm-thick axial slices. In the present work, using a $0.5 \mathrm{~mm}$ slice thickness with a thinner interval of reconstruction $(0.3 \mathrm{~mm})$ for MSCT and $0.25 \mathrm{~mm}$ voxel size for CBCT, 90.4\% and $90.7 \%$ of sensitivity and $96.3 \%$ and $95.9 \%$ of specificity (median values) were found regarding the number of simulated lesions respectively.

CT technology used for image manipulation and visualization of structures in multiple planes provides better identification of anatomical structures in the axial, coronal and sagittal planes, thus increasing image interpretation. The evaluation of complex diseases and conditions in the maxillofacial region requires more accurate image exams associated with multiplanar analysis for a complete delimitation and identification of the pathological process and a better understanding of the disease behavior. ${ }^{24}$ Currently, MPR and cross sectional images associated with tridimensional (3D) reconstruction represent the most useful imaging modalities for diagnoses and surgical planning in the oral and maxillofacial regions. ${ }^{13,14,16,24}$ Cross sectional images, extensively used for the planning of dental implants, were obtained from original CT images which were directed perpendicularly to a plane previously outlined, and depicted the anatomy better than straight sagittal planes, justifying the choice of that protocol in our research.

Utumi et al. ${ }^{14}$ Perrella et al. ${ }^{16}$ found differences of accuracy regarding mandibular lesions when they used, respectively, different post-processing images and different slice thicknesses from helical CT. Warnke et al. ${ }^{25}$ evaluated the applicability of a multiplanar reformatting program for delineation 
and quantitative evaluation of the temporomandibular joint in the sagittal and coronal planes. Those authors observed that the combination of different image visualization planes provided more accurate images than conventional CT. In their study, $100 \%$ of the pathological bone alterations were observed. Additionally, the use of reduced thicknesses in MSCT and smaller voxels in CBCT, as our work did, allows greater sensitivity and specificity, which corroborates previous studies.

Cara et al. ${ }^{15}$ compared the validity of different single- and multislice CT for analyses of simulated lesions in the head of the mandible. Sensitivity results were: axial single slice $(62.7 \%)$, axial multislice (66.2\%), axial/MPR single slice (72.7\%), axial/ MPR multislice (93.1\%). The association of axial images with MPR, using MSCT scans, demonstrated a higher accuracy than the single slice method. The present study determined the validity of images acquired by using MSCT and CBCT with different protocols (axial/MPR images and cross sectional slices). Our results showed that both observation protocols had significant sensitivity and specificity results in the detection of simulated bone lesions for both examiners compared to the gold standard, with no significant statistical difference (Table 3).

The association of CT protocols for visualization of simulated bone lesions was established with the aim of improving the visualization of the presence of mandibular bone alterations. Despite the high values of specificity and sensitivity found, cross sectional slices should be used together with the visu-

\section{References}

1. Ozen T, Kamburoğlu K, Cebeci AR, Yüksel SP, Paksoy CS. Interpretation of chemically created periapical lesions using 2 different dental cone-beam computerized tomography units, an intraoral digital sensor, and conventional film. Oral Surg Oral Med Oral Pathol Oral Radiol Endod. 2009 Mar;107(3):426-32.

2. Mahesh M. Search for isotropic resolution in CT from conventional through multiple-row detector. Radiographics. 2002 Jul-Aug;22(4):949-62.

3. Cavalcanti MG, Ruprecht A, Vannier MW. 3D volume rendering using multislice CT for dental implants. Dentomaxillofac Radiol. 2002 Jul;31(4):218-23. alization of axial, and MPR images. These simultaneous image analyses improve interpretation and prevent a misleading visualization due to flaws in the process of image reconstruction. It is our opinion that MSCT and CBCT may improve the results of early detection of bone lesions in vivo, since good sensitivity and specificity rates were obtained, even with tiny simulated lesions.

\section{Conclusion}

CBCT may be considered a valuable imaging tool for the identification of simulated bone lesions, showing results similar to those of MSCT. The cross sectional slices and axial/MPR images were highly accurate in the identification of simulated mandibular bone lesions (cortical bone destruction and number of the lesions in each mandible) proving to be useful for bone lesion diagnosis.

\section{Acknowledgements}

The authors would like to thank the São Paulo Research Foundation (FAPESP) for the financial support provided through grant no. 2005/02157-8 and PhD grant no. 2006/05251-8, and the Coordination for the Advancement of Higher Education Personnel (CAPES) for the financial support provided through a $\mathrm{PhD}$ grant. The authors are also grateful to Odonto X (Dr. Reinaldo Rosa), Rio de Janeiro, Brazil, where the CBCT images were produced, and to the Department of Anatomy of the Gama Filho University School of Medicine, Rio de Janeiro, Brazil, for providing the dry mandibles.

4. Guerrero ME, Jacobs R, Loubele M, Schutyser F, Suetens P, van Steenberghe D. State-of-the-art on cone beam CT imaging for preoperative planning of implant placement. Clin Oral Investig. 2006 Mar;10(1):1-7.

5. Scarfe WC, Farman AG, Sukovic P. Clinical applications of cone-beam computed tomography in dental practice. J Can Dent Assoc. 2006 Feb;72(1):75-80.

6. Moshiri M, Scarfe WC, Hilgers ML, Scheetz JP, Silveira AM, Farman AG. Accuracy of linear measurements from imaging plate and lateral cephalometric images derived from conebeam computed tomography. Am J Orthod Dentofacial Orthop. 2007 Oct;132(4):550-60. 
7. Hassan B, van der Stelt P, Sanderink G. Accuracy of threedimensional measurements obtained from cone beam computed tomography surface-rendered images for cephalometric analysis: influence of patient scanning position. Eur J Orthod. 2009 Apr;31(2):129-34.

8. Bernaerts A, Vanhoenacker FM, Hintjens J, Chapelle K, De Schepper AM. Imaging approach for differential diagnosis of jaw lesions: a quick reference guide. JBR-BTR. 2006 JanFeb;89(1):43-6.

9. Ludlow JB, Davies-Ludlow LE, Brooks SL, Howerton WB. Dosimetry of 3 CBCT devices for oral and maxillofacial radiology: CB Mercuray, New Tom 3G and i-CAT. Dentomaxillofac Radiol. 2006 Jul;35(4):219-26.

10. Cavalcanti MG, Haller JW, Vannier MW. Three-dimensional computed tomography landmark measurement in craniofacial surgical planning: experimental validation in vitro. J Oral Maxillofac Surg. 1999 Jun;57(6):690-4.

11. Schulze D, Blessmann M, Pohlenz P, Wagner KW, Heiland M. Diagnostic criteria for the detection of mandibular osteomyelitis using cone beam computed tomography. Dentomaxillofac Radiol. 2006 Jul;35(4):232-5.

12. Suomalainen A, Vehmas T, Kortesniemi M, Robinson S, Peltola J. Accuracy of linear measurements using dental cone beam and conventional multislice computed tomography. Dentomaxillofac Radiol. 2008 Jan;37(1):10-7.

13. Perrella A, Lopes PM, Rocha RG, Fenyo-Pereira M, Cavalcanti MG. Influence of dental metallic artifact from multislice CT in the assessment of simulated mandibular lesions. J Appl Oral Sci. 2010 Mar-Apr;18(2):149-54.

14. Utumi ER, Perrella A, Albuquerque MA, Adde CA, Rocha RG, Cavalcanti MG. Evaluation of simulated bone lesion in the head of the mandible by using multislice computed tomography. J Appl Oral Sci. 2009 Sep-Oct;17(5):521-6.

15. Cara AC, Gaia BF, Perrella A, Oliveira JX, Lopes PM, Cavalcanti MG. Validity of single- and multislice CT for assessment of mandibular condyle lesions. Dentomaxillofac Radiol. 2007 Jan;36(1):24-7.
16. Perrella A, Borsatti MA, Tortamano IP, Rocha RG, Cavalcanti MG. Validation of computed tomography protocols for simulated mandibular lesions: a comparison study. Braz Oral Res. 2007 Apr-Jun;21(2):165-9.

17. Naitoh M, Nakahara K, Suenaga Y, Gotoh K, Kondo S, Ariji E. Comparison between cone-beam and multislice computed tomography depicting mandibular neurovascular canal structures. Oral Surg Oral Med Oral Pathol Oral Radiol Endod. 2010 Jan;109(1):e25-31.

18. Pinsky HM, Dyda S, Pinsky RW, Misch KA, Sarment DP. Accuracy of three-dimensional measurements using cone-beam CT. Dentomaxillofac Radiol. 2006 Nov;35(6):410-6.

19. Mischkowski RA, Scherer P, Ritter L, Neugebauer J, Keeve E, Zöeller JE. Diagnostic quality of multiplanar reformations obtained with a newly developed cone beam device for maxillofacial imaging. Dentomaxillofac Radiol. 2008 Jan;37(1):1-9.

20. Dreiseidler T, Mischkowski RA, Neugebauer J, Ritter L, Zöeller JE. Comparison of cone-beam imaging with orthopantomography and computerized tomography for assessment in presurgical implant dentistry. Int J Oral Maxillofac Implants. 2009 Mar-Apr;24(2):216-25.

21. Baxter BS, Sorenson JA. Factors affecting the measurement of size and CT number in computed tomography. Invest Radiol. 1981 Jul-Aug;16(4):337-41.

22. Cavalcanti MGP, Santos DT, Perrella A, Vannier MW. CTbased analysis of malign tumor volume and localization. A preliminary study. Braz Oral Res. 2004 Oct-Dec;18(4):338-44.

23. Shaha AR. Preoperative evaluation of the mandible in patients with carcinoma of the floor of the mouth. Head Neck. 1991 Sep-Oct;13(5):398-402.

24. Ahmad M, Freymiller E. Cone Beam Computed Tomography: Evaluation of Maxillofacial Pathology. J Calif Dent Assoc. 2010 Jan;38(1):41-7.

25. Warnke T, Carls FR, Sailer HF. A new method for assessing the temporomandibular joint quantitatively by dental scan. J Craniomaxillofac Surg. 1996 Jun;24(3):168-72. 